\title{
Impacto económico del tratado de libre comercio y su relación con la competitividad de las pymes período 2014-2019, Olivos
}

\author{
Liz Maribel Robladillo Bravo \\ lizrobladillo.21@gmail.com \\ Universidad Nacional Federico Villarreal
}

\section{RESUMEN}

En el presente texto se pretende determinar la relación del impacto económico de los Tratados de libre Comercio con la Competitividad de las PYMES agroexportadoras del distrito de Los Olivos en Lima, durante el período 2014-2019. Realizando énfasis en el nivel de exportación, cumplimiento de normas consignadas en los acuerdo y la creación de empleo.

Palabras clave: tratado de libre comercio; competitividad; pymes 


\title{
Economic impact of the free trade agreement and its relationship with the competitiveness of SMEs 2014-2019 period, Olivos
}

\begin{abstract}
This text aims to determine the relationship of the economic impact of free trade agreements with the competitiveness of agro-exporting SMEs in the Los Olivos district in Lima, during the 2014-2019 period. Emphasizing the level of export, compliance with standards set forth in the agreement and job creation.
\end{abstract}

Key words: free trade agreement; competitiveness; SMEs

Artículo recibido: 02 noviembre. 2021 Aceptado para publicación: 28 noviembre 2021 Correspondencia: izrobladillo.21@gmail.com Conflictos de Interés: Ninguna que declarar 


\section{INTRODUCCIÓN}

Los países de América Latina han desarrollado estrategias para consolidar su participación en los mercados internacionales. Ello ha conllevado a reforzar las políticas de Estado en materia comercial, entre otras, el establecimiento de acuerdos comerciales regionales celebrados con naciones de la misma región o extrarregionales. En este contexto, se ha observado que las mayores beneficiarias de los acuerdos comerciales han sido aquellas empresas de gran tamaño, con conocimiento del mercado y con preparación para identificar y aprovechar las oportunidades que se les ofrecía. Cabe precisar que en Latinoamérica las grandes empresas son importantes actores económicos; sin embargo, son las pequeñas y medianas empresas los motores de las economías de la región, pues representan el $95 \%$ de las empresas constituidas y son la fuente del $67 \%$ del empleo.

Explicando la importancia de las PYMES en la dinamización de la economía peruana, así como la importancia y potencial de su internacionalización progresiva, en aras de un mayor aprovechamiento de los acuerdos comerciales. Así mismo saber los principales desafíos que estas enfrentan para internacionalizarse y ser competitivas en el marco los acuerdos comerciales. Es por ello la importancia del Plan Estratégico Nacional exportador y a su vez algunos beneficios particulares para las PYMES tras la firma de los acuerdos comerciales.

En países en vías de desarrollo como el Perú, las micro, pequeñas y medianas empresas representan un estrato importante en la estructura productiva del país, tanto por la cantidad de establecimientos como por la generación de empleo.

Es por ello la necesidad de trabajar en importantes puntos como la formalización, la digitalización, la investigación y desarrollo (I+D), el financiamiento, la productividad, el capital humano y sus capacidades. Estos son importantes aspectos por desarrollar que permitirán que el país se beneficie de manera plena, de la apertura económica y de los tratados de libre comercio, a través de la participación de las PYMES.

Por otro lado, muchas de las PYMES exportadoras en nuestro país no realizan una correcta evaluación de sus mercados de destino de exportación, siendo este uno de los principales motivos de fracaso en su participación, existen PYMES que no logran acceder a información exacta del mercado al cual aspiran exportar. En la mayoría de casos, no conocen las necesidades reales de sus clientes finales. Siendo así que, el intermediario quién se beneficia de las mayores ganancias por la venta del producto, pues éste si conoce 
al cliente final. Se ha comprobado que los márgenes de intermediación de varios de los distribuidores de productos latinoamericanos están entre los más altos del mundo, es decir buscar como compatibilizar las productividades de trabajo y capital en el tiempo mediante el impacto de los tratados de libre comercio y su relación con la competitividad.

\section{A continuación, se presentan algunos antecedentes acerca de la presente investigación:}

Tovar (2018), en su tesis, un Análisis Gravitacional de la Creación y Desviación Comercial en el marco del Tratado de Libre Comercio entre Perú-Estados Unidos. La presente investigación está orientada a comprobar si han existido efectos positivos traducidos en el aumento de los flujos comerciales en todas las secciones del sistema armonizado, a través de la medición de los efectos de creación y desviación comercial producidos por el TLC Perú-Estados Unidos, utilizando datos desagregados a 10 dígitos entre los años 1995-2015. Para ello, se utiliza la metodología planteada en Carrère (2006), MacPhee y Sattayanuwat (2014), Tumbarello (2007) y Yang y Martínez (2013) que utilizan tres variables dummy para medir estos efectos. El método de estimación que se utiliza es el llamado Pseudo Poisson Maximum Likelihood (PPML), introducido por Santos Silva y Tenreyro (2006). Entre los principales resultados se destaca que el TLC (sin distinciones entre secciones) genera efectos positivos, pero no significativos en el nivel de comercio. Asimismo, el TLC no ha generado un incremento en 7 de 21 secciones, debido a la existencia del efecto de desviación comercial intrabloque, mientras que sí presentó incrementos en las secciones 1 (animales vivos y productos del reino animal) y 18 (instrumentos y aparatos de óptica, fotografía o cinematografía; instrumentos y aparatos médico-quirúrgicos; aparatos de relojería; instrumentos musicales). Para el resto de secciones se obtuvieron resultados no significativos en la variable que mide el efecto del comercio intrabloque, pero sí presentaron resultados significativos para las variables que miden la creación o desviación comercial de importaciones y exportaciones. Por tal motivo, se realizan recomendaciones de políticas orientadas a revertir estos efectos para un mejor aprovechamiento de los acuerdos comerciales.

Chen Min (2017), la tesis tuvo como objetivo demostrar si el tratado de Libre Comercio (TLC) Perú - China, incide en las agroexportaciones a este país, dado que los Tratados son importantes para el crecimiento económico, así como para el fortalecimiento de las empresas que cada día tratan de mejorar en cuanto a su productividad, calidad, etc., buscando que sus productos tengan un mejor destino en las exportaciones. Con relación 
a la recopilación de la información del marco teórico, el aporte brindado por los especialistas relacionados con cada una de las variables: Tratado de Libre Comercio (TLC) y agroexportación, el mismo que clarifica el tema en referencia, así como también amplia el panorama de estudio con el aporte de los mismos; respaldado con el empleo de las citas bibliográficas que dan validez a la investigación. En suma, en lo concerniente al trabajo de campo, se encontró que la técnica e instrumento empleado, facilitó el desarrollo del estudio, culminando esta parte con la contrastación de las hipótesis y la discusión. Por último, los objetivos planteados en la investigación han sido alcanzados a plenitud, como también los datos encontrados en el estudio facilitaron el logro de los mismos. La conclusión principal del estudio fue que el tratado de Libre Comercio (TLC) Perú - China, incide favorablemente en las agroexportaciones a este país.

Camargo (2016), el objetivo general de la presente investigación consistió en evaluar el TLC Perú China y su impacto en la estructura del comercio exterior del Perú en el período 2010 - 2013. Desarrolla como objetivos específicos determinar cómo el TLC firmado con China ha impactado en la actividad de exportación del comercio exterior del Perú, evaluar si el nivel de las Importaciones desde China se mantiene o se ha incrementado con la firma del TLC, así como determinar y explicar si la actual Balanza Comercial, entre Perú y China, es deficitaria o arroja un superávit para el Perú. La tesis presenta un enfoque metodológico cuantitativo, aplicando la correlación entre Variables de Estudio a través de sus indicadores, y con diseño no experimental, Como resultado de la investigación a partir del análisis de la información procesada, se determina que hay una influencia negativa entre las variables de estudio del TLC, entre China y Perú. Se concluye que no hay inversión significativa de China en el Perú en Investigación y Desarrollo, que se necesita diversificar la producción para exportaciones de productos no tradicionales, que se requiere mayor explotación de los temas relativos a la cooperación minera e industrial, y que falta decisión para promover una agresiva política de mayor inversión China en el Perú.

Ferradas y Flores (2015), estudio descriptivo del tratado de libre comercio para mejora de la factibilidad de exportación de quinua orgánica a los estados unidos, ventajas y desventajas en el año 2015. La presente tesis estudio descriptivo del tratado de Libre Comercio para mejora de la factibilidad de exportación de quinua orgánica a los estados unidos, ventajas y desventajas en el año 2015; está orientada a la mejora de la factibilidad 
de exportación con el Tratado de libre comercio el cual sirve como una información oportuna que necesitan los productores y exportadores peruanos para ampliar su conocimiento sobre éste. Las barreras que se identifican son de carácter para-arancelario como son: permisos sanitarios, regulaciones de etiquetado, de calidad, etc. Asimismo, la consultoría aborda el tema del bioterrorismo y leyes concernientes a la importación de alimentos en Estados Unidos. El estudio presenta referencias e información clave de dicho mercado para las empresas peruanas. Se basa en una serie de entrevistas empresariales y visitas a instituciones públicas y privadas involucradas en el desarrollo del comercio y promoción de las exportaciones peruanas, las cuales aportan valiosa información de acceso de mercados que se utilizan a largo de todo el documento. En lo referente a las fuentes secundarias, se consideró los datos de estudios relacionados a la exportación de alimentos a Estados Unidos, fuentes oficiales como FDA, Comisión internacional de comercio de los Estados Unidos, etc. Según las teorías de "la nueva teoría del comercio de Paul Krugman (1979)", "la demanda recíproca de John Stuart Mill (1848)", el tratado de libre comercio garantiza el acceso preferencial de las exportaciones.

Calderón (2015). Análisis de la competitividad empresarial en las PYMES del sector del calzado del barrio el Restrepo de la ciudad de Bogotá, a partir del factor humano. La investigación mencionada busca analizar la competitividad empresarial de las PYMES del sector del calzado del barrio el Restrepo de la ciudad de Bogotá, a partir del estudio del capital humano y su contribución al mejoramiento de la competitividad empresarial de estas PYMES. Para ello se realizó una investigación cualitativa donde se entrevistaron en profundidad 25 personas entre las cuales se encuentran empresarios (as) y empleados (as) de estas organizaciones. Así mismo, se hizo un trabajo de campo mediante toma de fotografías, realización de bitácoras y notas de campo. Esta información cualitativa se procesó y analizó con la ayuda del programa de análisis de datos cualitativos Atlas Ti.7. La investigación parte en primer lugar de una revisión del estado del arte sobre las variables de estudio: competitividad y capital humano. En segundo lugar, se realiza una revisión a las políticas y planes de competitividad que el Gobierno Nacional ha realizado en los últimos dos periodos presidenciales. En tercer lugar, se explica la metodología empleada en la investigación, se analiza la información recolectada y se presentan los resultados. Finalmente se presentan una serie de propuestas para mejorar la 
competitividad de las PYMES del sector del calzado del barrio el Restrepo de la ciudad de Bogotá.

\subsection{Plantamiento del Problema}

El propósito de la presente investigación titulada "Impacto económico del Tratado de Libre Comercio y su relación con la Competitividad de las PYMES” consiste en analizar cómo han impactado o impactarán dichos tratados sobre las principales variables macroeconómicas de la economía del Perú como en sus exportaciones de los sectores notradicionales, es decir con cierto valor agregado, como son de las agroexportaciones (frutas y hortalizas); asimismo, se estudiará la generación de empleo productivo motivado por dichas exportaciones. En el caso del Tratado de Libre Comercio (TLC) con los Estados Unidos el análisis cubrirá el periodo 2014- 2019 debido a que se intentará evaluar adicionalmente el impacto de los antecedentes del TLC con dicho país como fueron el APTA y luego el APTDEA; en realidad el TLC entre el Perú y los Estados Unidos solamente vino a confirmar las ventajas del comercio internacional peruano hacia dicho país que ya se les había concedido vía los dos acuerdos anteriores mencionados; asimismo, en el análisis de la evolución del comercio peruano hacia los Estados Unidos se tomará en cuenta el crecimiento del enorme poder adquisitivo de la población estadounidense sobre las compras al Perú. Con relación al impacto del TLC firmado entre el Perú y China, debido a su reciente firma, se analizará el impacto que ha tenido en el corto plazo y sus perspectivas hacia el mediano plazo sobre las exportaciones peruanas hacia dicho país; en tal sentido el periodo de análisis será el 2014 -2019; de otro lado, al igual que en el caso de los Estados Unidos, también se evaluará el rápido crecimiento de la economía China durante el periodo mencionado y su impacto en sus compras de productos peruanos con valor agregado. Por otra parte, se estudiará en qué medida la estrategia económica del Perú y en particular sus políticas monetarias, cambiaria y de promoción de las exportaciones durante el periodo 2014-2019 han contribuido al esfuerzo exportador peruano como complemento a las firmas de los TLCs mencionados. Se espera que las conclusiones y recomendaciones del presente trabajo de investigación sean de utilidad para la mejora del buen gobierno y la transformación económica del país.

\subsection{Formulación del Problema}

\section{Problema General}

- ¿De qué manera el impacto económico del Tratado de libre Comercio se relaciona 
con la Competitividad de las PYMES agroexportadoras de los Olivos- Lima durante el período 2014-2019?

\section{Problemas Específicos}

- ¿Cómo se relaciona el nivel de exportación de productos agrícolas en el TLC con la Competitividad de las PYMES agroexportadoras de los Olivos- Lima durante el período 2014-2019?

- ¿Cómo se relaciona el cumplimiento de las normas del TLC con la Competitividad de las PYMES agroexportadoras de los Olivos- Lima durante el período 2014-2019?

- ¿Cómo se relaciona la creación de empleos derivados del TLC con la Competitividad de las PYMES agroexportadoras de los Olivos- Lima durante el período 2014-2019?

\subsection{Hipótesis}

\section{Hipótesis General}

- El impacto económico del Tratado de libre Comercio se relaciona con la Competitividad de las PYMES que afecta a la población de los Olivos-Lima durante el período 2014-2019.

\section{Hipótesis Específicas}

- El nivel de exportación de productos agrícolas en el TLC se relaciona de forma positiva con la Competitividad de las PYMES agroexportadoras de los Olivos- Lima durante el período 2014-2019.

- El cumplimiento de las normas del TLC se relaciona de forma positiva con la Competitividad de las PYMES agroexportadoras de los Olivos- Lima durante el período 2014-2019.

- La creación de empleos derivados del TLC se relaciona de forma positiva con la Competitividad de las PYMES agroexportadoras de los Olivos- Lima durante el período 2014-2019.

\section{METODOLOGÍA}

El tipo de investigación del estudio, es descriptivo correlacional. Es descriptivo: en cuanto se pretende especificar las propiedades, las características y los perfiles de personas, grupos, comunidades, y procesos que de lo que se investiga. Es de tipo correlacional, porque pretende responder si existen asociaciones entre las variables objeto de estudio.

\section{Este diseño se grafica de la siguiente manera:}




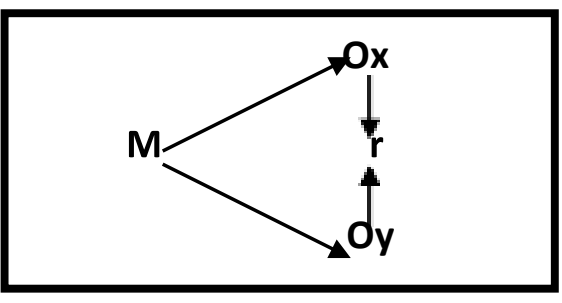

\section{En el esquema:}

$\mathrm{M} \quad=$ Muestra de investigación

$\mathrm{Ox}, \mathrm{Oy}=$ Observaciones de las variables

$\mathrm{r} \quad=$ Relaciones entre variables

\subsection{Población y muestra}

Población. La población estará conformada por 100 empresarios PYMES agroexportadores de los Olivos - Lima.

Muestra. Se determinó una muestra representativa y suficiente, en base a la siguiente fórmula:

- Muestra: "M"

- Población: "N" = 100 empresarios Pyme de los Olivos- Lima

- Z: coeficiente de confiabilidad, que de acuerdo a nuestra investigación es de

- 1.96 porque el nivel de confianza es de $95 \%$

- P: (0.5) proporción estimada de éxito asumida al 50\%

- Q: (0.5) proporción estimada de fracaso asumida al 50\%

- E: error absoluto o precisión, que en este caso se expresa en fracción de uno y es de 0.085

\section{Dónde:}

$$
\begin{aligned}
& \mathbf{M}=\frac{\mathrm{N}\left(\mathrm{Z}_{0.05}\right)^{2} \mathrm{P} . \mathrm{q}}{(\mathrm{N}-1)(\mathrm{E})^{2}+\left(\mathrm{Z}_{0.05}\right)^{2} \mathrm{p} . \mathrm{q}} \\
& \mathbf{M}=\frac{100(1.96)^{2}(0.5)^{2}}{(100-1)(0.085)^{2}+(1.96)^{2}(0.5)^{2}} \\
& \mathbf{M}=\frac{100(0.9604)}{(99)(0.085)^{2}+(1.96)^{2}(0.5)^{2}} \\
& \mathbf{M}=\frac{115.248}{1.67567} \quad \mathbf{M}=\mathbf{5 7 . 3 1}
\end{aligned}
$$


Finalmente se determinó una muestra de 57 empresas Pyme, como elemento de estudio para el desarrollo de la investigación.

\subsection{Operacionalización de variables}

\section{Cuadro 1}

Matriz de operacionalización de la variable Independiente

\begin{tabular}{|c|c|c|c|c|}
\hline Variables & Indicadores & Unidad de medida & Escala & $\begin{array}{l}\text { Valor } \\
\text { final }\end{array}$ \\
\hline \multirow{11}{*}{$\begin{array}{c}\text { Impacto } \\
\text { económico del } \\
\text { tratado de } \\
\text { libre comercio }\end{array}$} & \multirow{3}{*}{ Exportación } & Nivel de exportación & \multirow{5}{*}{$\begin{array}{l}\text { 1. Totalmente en } \\
\text { desacuerdo }\end{array}$} & \multirow{11}{*}{$\begin{array}{c}\text { Alto } \\
\text { Medio } \\
\text { Bajo }\end{array}$} \\
\hline & & Aranceles & & \\
\hline & & $\begin{array}{l}\text { Apertura de } \\
\text { mercados }\end{array}$ & & \\
\hline & \multirow{3}{*}{ Normas } & $\begin{array}{c}\text { Estabilidad y } \\
\text { predictibilidad }\end{array}$ & & \\
\hline & & Normas & & \\
\hline & & $\begin{array}{l}\text { Estándares de } \\
\text { calidad }\end{array}$ & $\begin{array}{l}\text { 3. Ni en acuerdo, ni } \\
\text { en desacuerdo }\end{array}$ & \\
\hline & \multirow{3}{*}{ Economía } & $\begin{array}{l}\text { Estabilidad de } \\
\text { entorno }\end{array}$ & \multirow{5}{*}{$\begin{array}{l}\text { 4. De acuerdo } \\
\text { 5. Totalmente de } \\
\text { acuerdo" }\end{array}$} & \\
\hline & & $\begin{array}{l}\text { Ambiente de } \\
\text { negocios }\end{array}$ & & \\
\hline & & $\begin{array}{l}\text { Crecimiento } \\
\text { económico }\end{array}$ & & \\
\hline & \multirow[t]{2}{*}{ Empleo } & Creación de empleos & & \\
\hline & & Bienestar & & \\
\hline
\end{tabular}

Fuente: Elaboración propia.

\subsection{Procedimientos}

Con el objetivo de analizar los datos recopilados en la investigación se empleó la estadística inferencial y descriptiva. Al respecto Valderrama (2015) señaló que luego de la recopilación de datos, se analizan los mismos para dar respuesta a la pregunta general y a la vez se pueda confirmar o rechazar la hipótesis de estudio. En este trabajo de indagación usamos la estadística inferencial y descriptiva con el objetivo de hacer un análisis estadístico de la recopilación de información.

En lo referente a la estadística descriptiva Hernández, Fernández y Baptista (2014) señala que el primer paso en la investigación es dar informar respecto de los valores o puntajes 
hallados en las variables. En lo que se refiere a la estadística inferencial Hernández, Fernández y Baptista (2014) señala que, para la comprobación de las hipótesis y las estimaciones de los parámetros en las variables, se utiliza la estadística.

\subsection{Análisis de datos}

Para recoger información sobre las variables del estudio se utilizará la técnica de la encuesta y como instrumento el cuestionario, en el cuál se evaluará la confiabilidad utilizando el índice de consistencia y coherencia interna de Alfa de Cronbach. Así mismo, el cuestionario fue evaluado por tres expertos, quienes concluirán si la encuesta CUMPLE REQUISITO DE APLICABILIDAD.

\section{RESULTADOS Y DISCUSIÓN}

\section{Hipótesis general}

- (Hi): El impacto económico del Tratado de libre Comercio se relaciona con la Competitividad de las PYMES que afecta a la población de los Olivos-Lima durante el período 2014-2019.

- (Ho): El impacto económico del Tratado de libre Comercio no se relaciona con la Competitividad de las PYMES que afecta a la población de los Olivos-Lima durante el período 2014-2019.

Nivel de significancia: $\alpha=0.05$ Estadístico: Rho de Spearman

Regla de decisión:

El nivel de significancia “p” es menor que $\alpha$, se rechaza H0.

El nivel de significancia " $p$ " es mayor que $\alpha$, no se rechaza H0

\section{Cuadro 2}

Correlación entre "Impacto económico del Tratado de libre Comercio" y "Competitividad de las PYMES"

\begin{tabular}{|c|c|c|c|c|}
\hline & & & $\begin{array}{c}\text { Impacto económico } \\
\text { del Tratado de libre } \\
\text { Comercio }\end{array}$ & $\begin{array}{c}\text { Competitiv } \\
\text { idad de las } \\
\text { PYMES }\end{array}$ \\
\hline \multirow{6}{*}{$\begin{array}{l}\text { Rho de } \\
\text { Spearman }\end{array}$} & \multirow{3}{*}{$\begin{array}{l}\text { Impacto económico } \\
\text { del Tratado de libre } \\
\text { Comercio }\end{array}$} & $\begin{array}{l}\text { Coeficiente de } \\
\text { correlación }\end{array}$ & 1,000 &, $667 * *$ \\
\hline & & Sig. (bilateral) & . & ,000 \\
\hline & & $\mathrm{N}$ & 57 & 57 \\
\hline & \multirow{3}{*}{$\begin{array}{l}\text { Competitividad de } \\
\text { las PYMES }\end{array}$} & $\begin{array}{l}\text { Coeficiente de } \\
\text { correlación }\end{array}$ &, $667 * *$ & 1,000 \\
\hline & & Sig. (bilateral) & ,000 & \\
\hline & & $\mathrm{N}$ & 57 & 57 \\
\hline
\end{tabular}

Fuente: Elaboración propia. 
En el cuadro 2, se observa que existe una relación fuerte entre "Impacto económico del Tratado de libre Comercio" y "Competitividad de las PYMES", con un coeficiente de correlación de 0.667 lo cual indicó una correlación moderada entre las variables y p = $0.000<0.01$ que corresponde a un nivel de confiabilidad del 99\%, por lo tanto se toma la decisión de rechazar la hipótesis nula y se acepta la hipótesis alterna; de tal forma que el impacto económico del Tratado de libre Comercio se relaciona con la Competitividad de las PYMES que afecta a la población de los Olivos-Lima durante el período 20142019 .

\section{Hipótesis Específica 1}

- (Hi): El nivel de exportación de productos agrícolas en el TLC se relaciona de forma positiva con la Competitividad de las PYMES agroexportadoras de los Olivos- Lima durante el período 2014-2019.

- (Ho): El nivel de exportación de productos agrícolas en el TLC no se relaciona con la Competitividad de las PYMES agroexportadoras de los Olivos- Lima durante el período 2014-2019.

Nivel de significancia: $\alpha=0.05 \quad$ Estadístico: Rho de Spearman

Regla de decisión:

El nivel de significancia “p” es menor que $\alpha$, se rechaza $\mathrm{H} 0$.

El nivel de significancia " $p$ " es mayor que $\alpha$, no se rechaza H0

\section{Cuadro 3}

Correlación entre "Nivel de exportación de productos agrícolas en el TLC" y

\section{"Competitividad de las PYMES"}

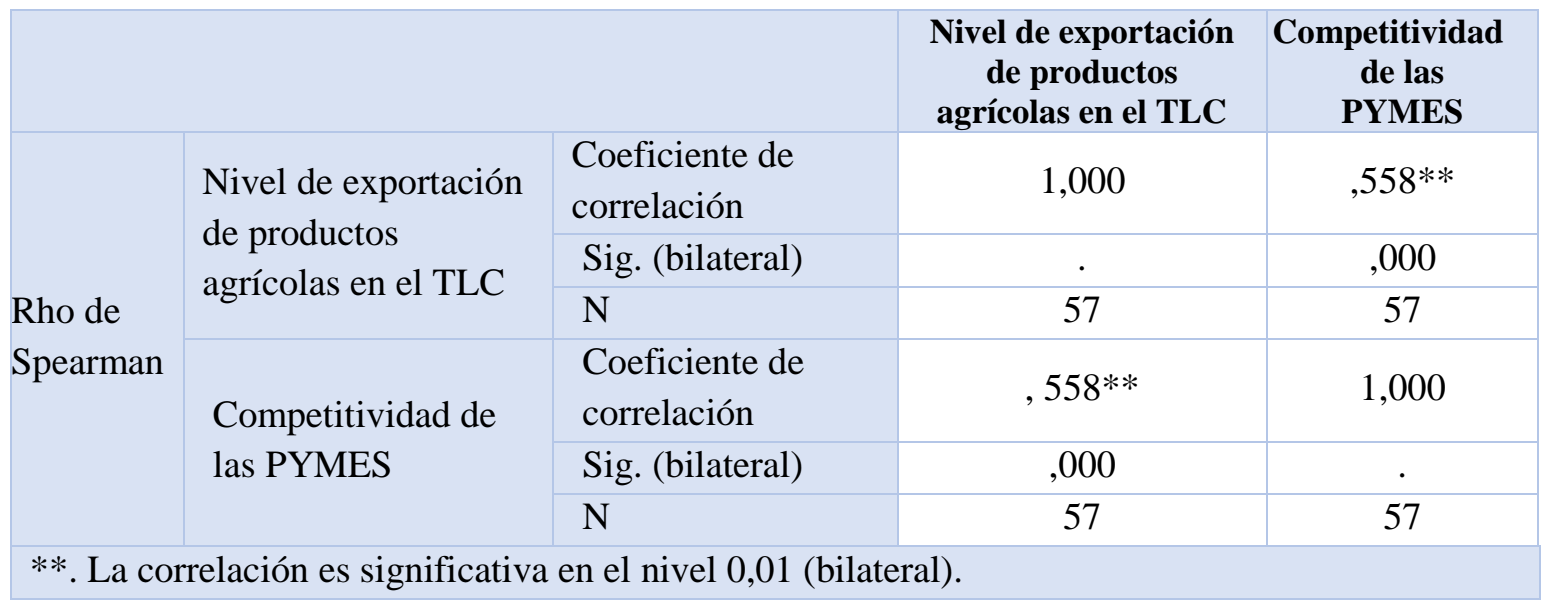

Fuente: Elaboración propia. 
En el cuadro 3, se observa que existe una relación fuerte entre "Nivel de exportación de productos agrícolas en el TLC" y "Competitividad de las PYMES", con un coeficiente de correlación de 0.558 lo cual indicó una correlación moderada entre las variables y p = $0.000<0.01$ que corresponde a un nivel de confiabilidad del 99\%, por lo tanto, se toma la decisión de rechazar la hipótesis nula y se acepta la hipótesis alterna; de tal forma que el nivel de exportación de productos agrícolas en el TLC no se relaciona con la Competitividad de las PYMES agroexportadoras de los Olivos- Lima durante el período 2014-2019.

\section{Hipótesis Específica 2}

- (Hi): El cumplimiento de las normas del TLC se relaciona de forma positiva con la Competitividad de las PYMES agroexportadoras de los Olivos- Lima durante el período 2014-2019.

- (Ho): El cumplimiento de las normas del TLC no se relaciona con la Competitividad de las PYMES agroexportadoras de los Olivos- Lima durante el período 2014-2019.

Nivel de significancia: $\alpha=0.05 \quad$ Estadístico: Rho de Spearman

Regla de decisión:

El nivel de significancia " $p$ ” es menor que $\alpha$, se rechaza $\mathrm{H} 0$.

El nivel de significancia " $p$ ” es mayor que $\alpha$, no se rechaza $\mathrm{H} 0$

\section{Cuadro 4}

Correlación entre "Cumplimiento de las normas del TLC" y "Competitividad de las PYMES”

\begin{tabular}{|c|c|c|c|c|}
\hline & & & $\begin{array}{l}\text { Nivel de exportación } \\
\text { de productos agrícolas } \\
\text { en el TLC }\end{array}$ & $\begin{array}{c}\text { Competitividad } \\
\text { de las } \\
\text { PYMES }\end{array}$ \\
\hline \multirow{6}{*}{$\begin{array}{l}\text { Rho de } \\
\text { Spearman }\end{array}$} & \multirow{3}{*}{$\begin{array}{l}\text { Nivel de exportación } \\
\text { de productos } \\
\text { agrícolas en el TLC }\end{array}$} & $\begin{array}{l}\text { Coeficiente de } \\
\text { correlación }\end{array}$ & 1,000 &, $643 * *$ \\
\hline & & Sig. (bilateral) & . &, 000 \\
\hline & & $\mathrm{N}$ & 57 & 57 \\
\hline & \multirow{3}{*}{$\begin{array}{l}\text { Competitividad de } \\
\text { las PYMES }\end{array}$} & $\begin{array}{l}\text { Coeficiente de } \\
\text { correlación }\end{array}$ &, $643 * *$ & 1,000 \\
\hline & & Sig. (bilateral) & ,000 & $\cdot$ \\
\hline & & $\mathrm{N}$ & 57 & 57 \\
\hline
\end{tabular}

Fuente: Elaboración propia.

En el cuadro 4, se observa que existe una relación fuerte entre "Cumplimiento de las normas del TLC" y "Competitividad de las PYMES", con un coeficiente de correlación 
de 0.643 lo cual indicó una correlación moderada entre las variables y p $=0.000<0.01$ que corresponde a un nivel de confiabilidad del 99\%, por lo tanto, se toma la decisión de rechazar la hipótesis nula y se acepta la hipótesis alterna; de tal forma que el cumplimiento de las normas del TLC se relaciona de forma positiva con la Competitividad de las PYMES agroexportadoras de los Olivos- Lima durante el período 2014-2019.

\section{Hipótesis Específica 3}

- (Hi): La creación de empleos derivados del TLC se relaciona de forma positiva con la Competitividad de las PYMES agroexportadoras de los Olivos- Lima durante el período 2014-2019.

- (Ho): La creación de empleos derivados del TLC no se relaciona con la Competitividad de las PYMES agroexportadoras de los Olivos- Lima durante el período 2014-2019.

Nivel de significancia: $\alpha=0.05 \quad$ Estadístico: Rho de Spearman

Regla de decisión:

El nivel de significancia “p” es menor que $\alpha$, se rechaza $\mathrm{H} 0$.

El nivel de significancia " $p$ ” es mayor que $\alpha$, no se rechaza $\mathrm{H} 0$

\section{Cuadro 5}

Correlación entre "creación de empleos derivados del TLC" y "Competitividad de las PYMES”

\begin{tabular}{|l|l|l|c|c|}
\hline & & & $\begin{array}{c}\text { Nivel de exportación } \\
\text { de productos } \\
\text { agrícolas en el TLC }\end{array}$ & $\begin{array}{c}\text { Competitividad } \\
\text { de las } \\
\text { PYMES }\end{array}$ \\
\hline & $\begin{array}{l}\text { Nivel de exportación } \\
\text { de productos } \\
\text { agrícolas en el TLC }\end{array}$ & $\begin{array}{l}\text { Coeficiente de } \\
\text { correlación }\end{array}$ & 1,000 &, $622^{* *}$ \\
\hline $\begin{array}{l}\text { Rho de } \\
\text { Spearman }\end{array}$ & Sig. (bilateral) &. &, 000 \\
\hline & $\begin{array}{l}\text { Competitividad de } \\
\text { las PYMES }\end{array}$ & $\begin{array}{l}\text { Coeficiente de } \\
\text { correlación }\end{array}$ &, $622^{* *}$ & 57 \\
\hline & Sig. (bilateral) &, 000 & 1,000 \\
\hline **. La correlación es significativa en el nivel 0,01 (bilateral). & & 57 & 57 \\
\hline
\end{tabular}

Fuente: Elaboración propia.

En el cuadro 5, se observa que existe una relación fuerte entre "creación de empleos derivados del TLC" y "Competitividad de las PYMES", con un coeficiente de correlación de 0.622 lo cual indicó una correlación moderada entre las variables y p $=0.000<0.01$ que corresponde a un nivel de confiabilidad del 99\%, por lo tanto, se toma la decisión de 
rechazar la hipótesis nula y se acepta la hipótesis alterna; de tal forma que la creación de empleos derivados del TLC se relaciona de forma positiva con la Competitividad de las PYMES agroexportadoras de los Olivos- Lima durante el período 2014-2019.

\section{CONCLUSIONES}

Se concluye que existe una relación fuerte entre "Impacto económico del Tratado de libre Comercio" y "Competitividad de las PYMES", con un coeficiente de correlación de 0.667 lo cual indicó una correlación moderada entre las variables y $\mathrm{p}=0.000<0.01$ que corresponde a un nivel de confiabilidad del 99\%, por lo tanto se toma la decisión de rechazar la hipótesis nula y se acepta la hipótesis alterna; de tal forma que el impacto económico del Tratado de libre Comercio se relaciona con la Competitividad de las PYMES que afecta a la población de los Olivos-Lima durante el período 2014-2019.

Se concluye que existe una relación fuerte entre "Nivel de exportación de productos agrícolas en el TLC" y "Competitividad de las PYMES", con un coeficiente de correlación de 0.558 lo cual indicó una correlación moderada entre las variables y p = $0.000<0.01$ que corresponde a un nivel de confiabilidad del $99 \%$, por lo tanto, se toma la decisión de rechazar la hipótesis nula y se acepta la hipótesis alterna; de tal forma que el nivel de exportación de productos agrícolas en el TLC no se relaciona con la Competitividad de las PYMES agroexportadoras de los Olivos- Lima durante el período 2014-2019.

Se concluye que existe una relación fuerte entre "Cumplimiento de las normas del TLC" y "Competitividad de las PYMES", con un coeficiente de correlación de 0. 643 lo cual indicó una correlación moderada entre las variables y p $=0.000<0.01$ que corresponde a un nivel de confiabilidad del $99 \%$, por lo tanto, se toma la decisión de rechazar la hipótesis nula y se acepta la hipótesis alterna; de tal forma que el cumplimiento de las normas del TLC se relaciona de forma positiva con la Competitividad de las PYMES agroexportadoras de los Olivos- Lima durante el período 2014-2019.

Se concluye que existe una relación fuerte entre "creación de empleos derivados del TLC" y "Competitividad de las PYMES", con un coeficiente de correlación de 0. 622 lo cual indicó una correlación moderada entre las variables y p $=0.000<0.01$ que corresponde a un nivel de confiabilidad del $99 \%$, por lo tanto, se toma la decisión de rechazar la hipótesis nula y se acepta la hipótesis alterna; de tal forma que la creación de empleos 
derivados del TLC se relaciona de forma positiva con la Competitividad de las PYMES agroexportadoras de los Olivos- Lima durante el período 2014-2019.

\section{REFERENCIAS BIBLIOGRÁFICAS}

Barboza, A. (2016). La rentabilidad en la micro y pequeñas empresas, del sector servicio - rubro tueste, molido y empaque de café, del Distrito de Villa Ricas, Provincia de Oxapampa, Departamento de Pasco, periodo 2015 (Tesis de licenciatura). Recuperada de http://repositorio.uladech.edu.pe /bitstream/handle/123456789/1079/RENTABILIDAD_MYPE_BARBOZA_D ECADA_ANGIE_DEL_PILAR.pdf?sequence $=1 \&$ isAllowed $=\mathrm{y}$

Billene, R. (2000). Análisis de Costos II. Recuperado de https://books.google. com.pe/books?id=L115rPanPqUC\&printsec $=$ frontcover $\& \mathrm{dq}=$ analisis $+\mathrm{de}+\mathrm{co}$ stos+II\&hl=es419\&sa=X\&ved=0ahUKEwiirYOpqOvXAhVI6SYKHZhWD4kQ 6a EIJTAA\#v=onepage $\& \mathrm{q}=$ analisis $\% 20 \mathrm{de} \% 20 \cos t$ os $\% 20 \mathrm{II} \& \mathrm{f}=\mathrm{false}$

Carrasco, S. (2008). Metodología de la Investigación Científica. (2.a ed.). Perú: San Marcos.

Cartier, E. (s.f.). ¿Cómo enseñar a determinar costos? Un problema no resuelto. Chivilcoy, Argentina.

Cautrecasas, L. (2012). Organización de la producción y dirección de Operaciones. Recuperado de https://books.google.com.pe/books/abo ut/ Organizaci\%C3\%B3n_de_la_producci\%C3\%B3n_y_direcc.html?id=6jNY 9FcLGcoC

Hernández, R., Fernández, C., y Baptista, P. (2014). Metodología de la Investigación. (5.a ed.). Mexico: McGra Hill.

Instituto Nacional de Estadística e Informática. (2012). Guía estadística para análisis de la información. Recuperado de http://censos.inei.gob.pe/Cen agro/redatam/doc/Guia\%20Estadistica.pdf

Junta Nacional del café. (Mayo, 2014). Producción cafetera enfrenta su mayor crisis de la última década, según JNC. Diario Gestión. Recuperado de https://gestion.pe/economia/produccion-cafetalera-enfrenta-mayor-crisis- ultimadecada-jnc-60587 
Macanas, R. (2015) Augmented Gravity Model of International Trade: An Empirical Application to ASEAN Intra and Extra-Regional Trade of Textiles and Clothing. SSRN. Journal of Economic Integration. Vol. 29, No. 1, March, pp. 64 - 94.

Macphee, C.y Wanasin S. (2014). Consequence of Regional Trade Agreements to Developing Countries.

Giovanni M. y Rodriguez-Clare A.( 1998). The Value of Trade Agreements in Presence of Political Pressures. Journal of Political Economy, 106(3), pp. 574 - 601.

Márquez-Ramos, L., Inmaculada Martínez-Zarzoso, I. y Suárez-Burguet, C. (2007). The Role of Distance in Gravity Regressions: Is There Really a Missing Globalization Puzzle?. Journal of Economic Analysis \& Policy. 7 (1), pp. 1-25.

Milton, S. y Siddique M.(2014). Trade creation and diversion under the ThailandAustralia Free Trade Agreement (TAFTA). Discussion Paper 14.26. University of Western Australia - Business School

Muñoz, L. y Gallegos, M. (2016). Programa de sensibilización para la producción de café orgánico en el distrito de La Peca departamento de Amazonas Perú (Tesis de licenciatura). Recuperada de http://repositorio.upao.edu.pe/bitstream/upaorep/1445/1/Mu\%C3\%B1oz_Leanita _Programa\%20_Sen sibilizacion_Produccion.pdf

Nahuamel, J. (2013). Competitividad de la Cadena Productiva del Café Orgánico en la Provincia de La Convención, Región Cusco (Tesis de magister). Recuperada de http://repositorio.lamolina.edu.pe/bitstream/handle/UNA IM/1098/E16-N3T.pdf? sequence $=3 \&$ isAllowed $=\mathrm{y}$

Organización Internacional del café. (2017). Infogram Perú. https://infogram.com/_/sp196ccwuxHaGfrNChky

Patiño, A. y Vergara, V. (Febrero, 2011). Producción orgánica de café, como alternativa a la masiva venta de terrenos productivos en tierras altas de Chiriquí. http://es.calameo.com/read/000327026e863d3 78d0d1

Siegel, S. (1980). Estadísticas no paramétricas Aplicadas a las Ciencias de la Conducta. México: Trillas.

Sistema Integrado de Estadísticas Agrarias. (s.f.). Estadística Agrícola. http://siea.minagri.gob.pe/siea/sites/default/files/ii_estad\%

C3\%ADstica_agricola.pdf 
Trademap. (2016). Lista de los mercados importadores para un producto exportado por Perú en 2016. http://www.trademap.org/ Country_SelProduct Country.aspx?nvpm=3|604||||090111|||6|1|1|2|1||2|1|1

Torres, M. (2009) Módulo de investigación. UNFV. 
6. ANEXOS (EN CASO DE QUE EXISTAN)

\section{Anexo 1 - Cuestionario Tratado de Libre Comercio}

\section{"IMPACTO ECONÓMICO DEL TRATADO DE LIBRE COMERCIO Y SU RELACIÓN CON LA COMPETITIVIDAD DE LAS PYMES PERÍODO 2014- 2019, OLIVOS"}

Objetivo: $\quad$ Establecer la relación que existe entre el impacto económico del Tratado de libre Comercio y su relación con la Competitividad de las PYMES que afecta a la población de los Olivos-Lima durante el período 20142019

Instrucciones: Los siguientes enunciados se utilizan con fines científicos, por favor conteste de forma cuidadosa y sincera marcando su respuesta con una cruz o aspa.

I. Datos del encuestado:

1. Sexo: Femenino ( ) Masculino ( )

2. Edad: 18 a $30($ ) $\quad 31$ a $50($ ) $51 \mathrm{a}+($ )

3. Cargo:

II. En cada enunciado, marcando con una cruz o aspa indicando si está:

- Totalmente en desacuerdo $\quad=1$

- En desacuerdo $=2$

- $\mathrm{Ni}$ de acuerdo ni en desacuerdo $=3$

- De acuerdo $=4$

- Totalmente de acuerdo $=5$ 


\section{Exportación}

1. El TLC ha propiciado el incremento la exportación de productos agrícolas

2. El TLC ha propiciado la reducción de los aranceles

3. El TLC ha propiciado la reducción y/o eliminación de obstáculos al ingreso de la agroexportación

4. Con el TLC encuentra facilidades para la apertura del mercado y acceso preferencial a los productos de agroexportación

5. Con el TLC ha aumentado la comercialización de productos nacionales

6. Los productos agropecuarios se puedan vender en el exterior sin aranceles ni trabas administrativas.

\section{Normas}

7. El TLC ha brindado estabilidad y predictibilidad en las reglas de juego del comercio de productos agrícolas

8. El TLC ha propiciado el establecimiento de normas que mejoran los términos comerciales

9. El TLC ha promovido el funcionamiento eficiente, transparente y ágil de las aduanas

10. El TLC ha promovido agilidad en los trámites aduaneros

11. Las Pymes se han adecuado a los estándares de calidad para la agroexportación

12. Las Pymes han desarrollado políticas internas de desarrollo y adecuación logística

Economía

13. El Tratado de Libre Comercio es beneficioso para la economía del país

14. El TLC ha propiciado un entorno estable y sin barreras para el comercio y la inversión

15. El TLC brindar a los inversionistas un ambiente de negocios más estable y seguro.

\section{Empleo}

16. El TLC ha propiciado la creación de empleos derivados de una mayor actividad exportadora

17. El TLC mejora el bienestar de la población a través de mejores empleos y un mayor crecimiento económico 
Anexo 2 - Cuestionario Competitividad

\section{"IMPACTO ECONÓMICO DEL TRATADO DE LIBRE COMERCIO Y SU RELACIÓN CON LA COMPETITIVIDAD DE LAS PYMES PERÍODO 2014- 2019, OLIVOS"}

Objetivo: $\quad$ Establecer la relación que existe entre el impacto económico del Tratado de libre Comercio y su relación con la Competitividad de las PYMES que afecta a la población de los Olivos-Lima durante el período 20142019

Instrucciones: Los siguientes enunciados se utilizan con fines científicos, por favor conteste de forma cuidadosa y sincera marcando su respuesta con una cruz o aspa.

III. Datos del encuestado:

1. Sexo: Femenino ( ) Masculino ( )

2. Edad: 18 a $30($ ) $\quad 31$ a $50($ ) $51 \mathrm{a}+($ )

3. Cargo:

IV. En cada enunciado, marcando con una cruz o aspa indicando si está:
- Bajo
$=1$
- Medio
$=2$
- Alto
$=3$

\begin{tabular}{|l|l|l|l|l|l|}
\hline Competitividad & & & & & \\
\hline Nivel de planificación estratégico & & & & & \\
\hline Nivel de capacidad financiera & & & & & \\
\hline Nivel de conocimiento del mercado & & & & & \\
\hline Capacidad del uso de TICS & & & & & \\
\hline Nivel de relacionamiento con empresas internacionales & & & & & \\
\hline Nivel de capacitación & & & \\
\hline
\end{tabular}

